OPEN ACCESS

Edited by:

Jorge L. Alio,

Miguel Hernández University, Spain

Reviewed by:

Darren Gregory,

University of Colorado, United States

Giulio Ferrari,

San Raffaele Hospital (IRCCS), Italy

*Correspondence:

Chie Sotozono

csotozon@koto.kpu-m.ac.jp

Specialty section:

This article was submitted to

Ophthalmology

a section of the journal

Frontiers in Medicine

Received: 22 January 2021

Accepted: 17 June 2021

Published: 28 July 2021

Citation:

Sotozono C, Ueta M and Kinoshita S

(2021) Japan: Diagnosis and

Management of Stevens-Johnson

Syndrome/Toxic Epidermal Necrolysis

With Severe Ocular Complications.

Front. Med. 8:657327.

doi: 10.3389/fmed.2021.657327

\section{Japan: Diagnosis and Management of Stevens-Johnson Syndrome/Toxic Epidermal Necrolysis With Severe Ocular Complications}

\author{
Chie Sotozono $^{1 *}$, Mayumi Ueta ${ }^{1}$ and Shigeru Kinoshita ${ }^{2}$ \\ ${ }^{1}$ Department of Ophthalmology, Kyoto Prefectural University of Medicine, Kyoto, Japan, ${ }^{2}$ Department of Frontier Medical \\ Science and Technology for Ophthalmology, Kyoto Prefectural University of Medicine, Kyoto, Japan
}

In 2005, the "Japanese Research Committee on Severe Cutaneous Adverse Reaction" (J-SCAR) presented the official "Diagnostic Criteria" for SJS/TEN, and the specific ocular findings are included in these very important criteria. In SJS/TEN cases involving ocular disorder, conjunctivitis often occurs prior to the onset of the high fever. In a Japanese survey, ocular involvement was observed in $77 \%$ of the cases, and the incidence of ocular sequelae increased depending on the score of the acute ocular severity findings. Pseudo-membrane formation and epithelial defects are considered to be high-risk signs of ocular sequelae. At the chronic stage, limbal stem cell deficiency, visual disturbance, and severe dryness of the ocular surface are the primary disease characteristics. In 2002, we started performing Cultivated Oral Mucosal Epithelial Transplantation (COMET) for the treatment of severe ocular disorders, including SJS/TEN. As an additional treatment method, we developed a new type of rigid contact lens (CL) that is 13 to $14.0-\mathrm{mm}$ in diameter, known as the "Limbal Rigid Contact Lens (Limbal CL)." Our Limbal Rigid CL greatly enhances the postoperative outcome of COMET. The detection rate of ocular surface bacteria is high in SJS/TEN cases. Thus, appropriate use of topical antibiotics reduces the risk of ocular surface inflammation. Moreover, rebamipide is an ophthalmic solution for dry eye that was developed in Japan, and it also has the effect of suppressing ocular surface inflammation. From disease onset until the chronic stage, the control of inflammation and stem cell loss is key to successfully treating eyes afflicted with SJS/TEN.

Keywords: Stevens-Johnson syndrome, toxic epidermal necrolysis, cultivated oral mucosal epithelial transplantation, Limbal-Rigid contact lens, Japan

\section{INTRODUCTION}

Stevens-Johnson syndrome (SJS), and its more severe variant, toxic epidermal necrolysis (TEN), are acute systemic disorders that can affect anyone, and at any age (1-3). A variety of drugs can be the cause of SJS/TEN. At the onset of the disease, a definitive diagnosis of SJS and TEN is often complex and confusing. 
In Japan, the "Japanese Research Committee on Severe Cutaneous Adverse Reaction" (J-SCAR) has been conducting diligent and extensive work on the diagnosis and treatment of SJS/TEN over the past two decades. In 2005, J-SCAR presented the official "Diagnostic Criteria and Systemic Severity Index Score" for SJS/TEN (4). Since then, SJS/TEN has been diagnosed based on these criteria. Importantly, the disease-specific ocular characteristics are now included as one of the supportive findings, and these criteria has enabled ophthalmologists to be a valued clinical "team member" for the diagnosis and treatment of SJS/TEN at the acute stage.

Both SJS and TEN are systemically self-limited lasting 68 weeks after onset. However, in SJS/TEN cases with severe ocular complications (SOC), persistent epithelial defects (PED) on the ocular surface can linger, ultimately resulting in ulceration and perforation (5). Finally, visual impairment and severe dryness of the eye remain as ocular sequelae $(6,7)$. At present, there is no standardized treatment strategy for SJS/TEN-related blindness worldwide. Patients with SJS or TEN require life-long management for ocular discomfort and morbidity. Our group has now developed both surgical and non-surgical therapeutic methods for the successful treatment of SJS/TEN.

In this review article, we describe the current diagnostic and therapeutic strategies for SJS/TEN with SOC now practiced in Japan, and provide a detailed summary of the multiyear clinical research and comprehensive Japanese survey on SJS/TEN conducted by Kyoto Prefectural University of Medicine, Kyoto, Japan.

\section{SJS/TEN WITH OCULAR INVOLVEMENT AT THE ACUTE STAGE}

At disease onset, ocular involvement in SJS/TEN is often easily overlooked due to the serious general symptoms and high lethality. Thus, the clinical characteristics of SJS/TEN with SOC need to be well-understood for early diagnosis and successful treatment.

\section{Characteristic Findings}

Our findings revealed that among 94 SJS/TEN patients with SOC, 75 (82\%) experienced common cold-like symptoms (general malaise, slight fever, sore throat, etc.) that preceded the skin eruptions. In all patients, except one, the disease was accompanied by very high fever (above $39^{\circ} \mathrm{C}$ ) at onset. Acute conjunctivitis and oral involvement (blisters, erosions, and bleeding of the mouth and lips) occurred in all patients who could recollect their symptoms in detail. Fingernail loss at the acute stage or deformation at presentation existed in all patients, thus suggesting that paronychia occurred in all patients at the acute phase (Figures $\mathbf{1 A - C}$ ). Other mucous-membrane involvements included those of the pharynx, respiratory tract, or ear canal (8). Forty-two patients reported episodes of acute conjunctivitis from several hours to 4 days prior to the skin eruptions, and 21 patients reported that skin eruptions and conjunctivitis occurred simultaneously. Only 1 patient reported the occurrence of conjunctivitis post skin eruption. All patients reported remembering their eye symptoms such as bilateral red eye or ocular pain at disease onset. Surprisingly, 11 patients were diagnosed with acute conjunctivitis by ophthalmologists prior to the development of systemic eruptions. Thus, ophthalmologists should be aware that acute conjunctivitis can occur prior to skin eruptions (8).

\section{Acute Ocular Severity Score}

Typically, pseudomembrane formation with corneal and/or conjunctival erosions occurs at the acute stage of SJS/TEN. Hence, we speculated that inflammation and epithelial defects are key aspects of the ocular findings at the acute stage and developed the "Acute Ocular Severity Score" on a scale from 0 to 3 (none, mild, severe, and very severe) according to the existence of hyperemia, corneal or conjunctival epithelial defect, and pseudomembrane formation (Figures 1D-F) (4). In a Japanese survey, ocular involvement was seen in $104(77 \%)$ of 135 SJS/TEN cases (87 SJS and 48 TEN cases arising between 2005 and 2007), and pseudomembrane formation and/or epithelial defects were seen in 62 of those cases (46\%) (4). The incidence of ocular sequelae increased depending on the score of the acute ocular severity. Thus, pseudomembrane formation and epithelial defects are considered to be high-risk signs of ocular sequelae.

As shown in our previous study, patient age and NSAIDs or cold remedies as the exposed drugs were the predictive factors for the increase of acute ocular severity (4) (Table 1). In our previously published retrospective studies on SJS/TEN cases at the chronic phase, mean patient age was between 20 and 30 years at disease onset and cold remedies were the exposed drugs that were used in more than $24 \%$ of the cases $(7,8)$ (Table 2 ).

\section{Treatment Strategy}

The use of systemic corticosteroids for acute SJS and TEN is controversial (9-11). Thus, the timing and dose of the administered steroid may be key to obtaining beneficial outcomes.

As a prospective study, we used systemic steroid-pulse and topical betamethasone treatments in five cases diagnosed within 4 days from disease onset (11). All 10 eyes successfully healed without visual dysfunction. In our retrospective analysis, visual prognoses were significantly better in the group receiving topical steroids at the acute stage compared with the no-treatment group (8). In the Japanese treatment guideline, steroid-pulse therapy and topical betamethasone have been recommended in SJS/TEN cases with severe ocular involvement; i.e., an Acute Ocular Severity Score of 2 or 3. In tears obtained from SJS cases at the acute stage with pseudomembrane and epithelial defects, IL-6, IL-8, and MCP-1 were found to be dramatically upregulated (12). Thus, it is important to suppress the ocular surface inflammation. Moreover, initiating treatment with systemic and topical steroids from the onset of the disease appears to be important for the improvement of the visual prognosis.

\section{Subacute Phase With PED}

PEDs occurring in the subacute phase of SJS/TEN are very difficult to treat. Massive inflammation on the ocular surface is often uncontrollable, even with the use of systemic and/or local 

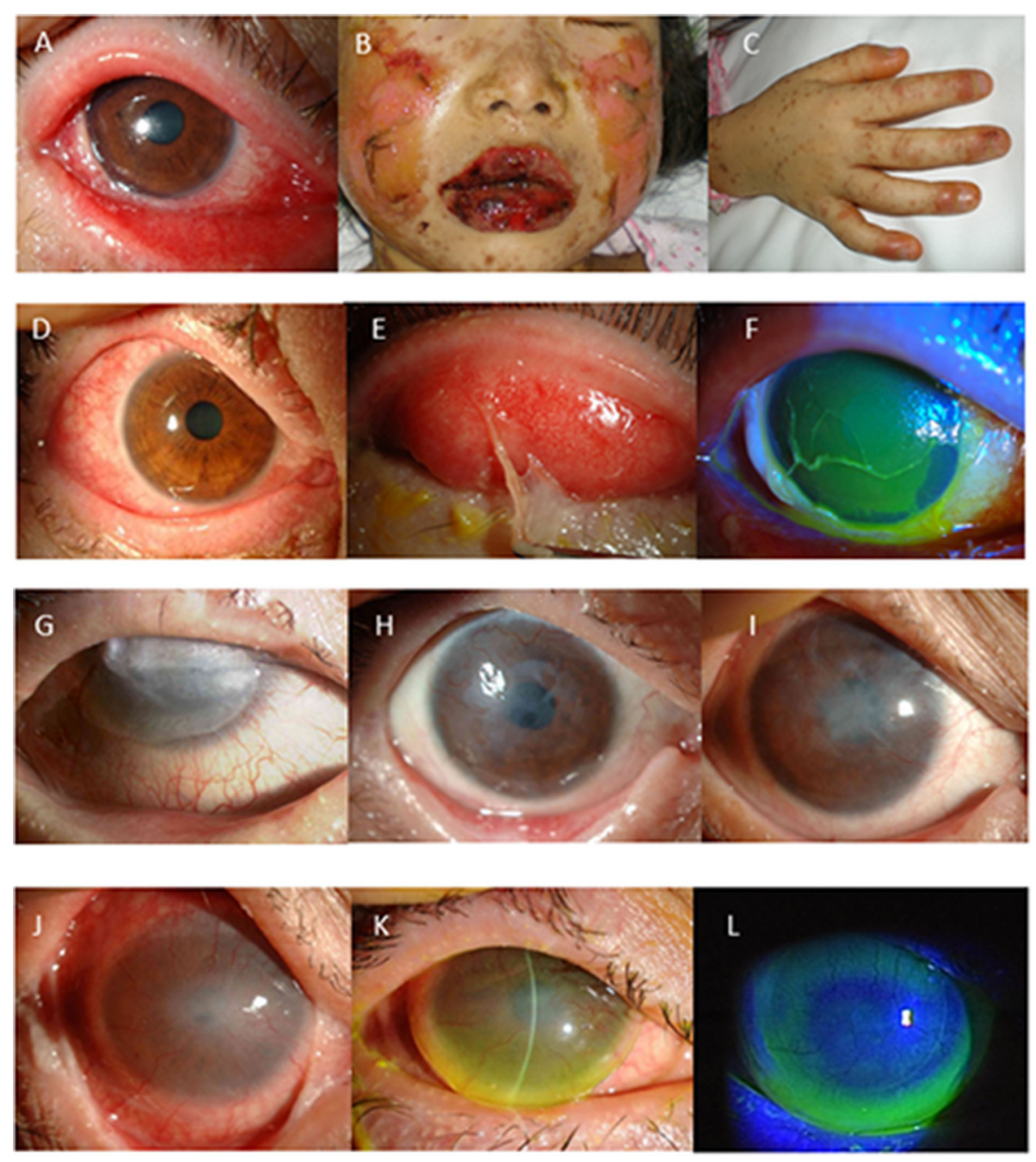

FIGURE 1 | (A-F) Representative photographs showing Stevens-Johnson syndrome/toxic epidermal necrolysis (SJS/TEN) at disease onset with severe ocular complications (SOC). (A) Conjunctivitis, which was accompanied by extensive loss of corneal and conjunctival epithelium. (B) Swollen and crusted lips. (C) Paronychia. In 66\% of the patients, conjunctival hyperemia (D) preceded skin eruption. Pseudomembrane formation (E) and corneal or conjunctival epithelial defect (F) develop at the acute stage. (G-I) Long-term effect of cultivated oral mucosal epithelial transplantation (COMET). Images of SJS/TEN cases obtained at before (G) and at 2-years $\mathbf{( H )}$ and 5-years postoperative (I). (J-L) Use of a limbal-supported rigid-type contact lens (Limbal-Rigid CL; Sun Contact Lens) for the treatment of SJS/TEN with ocular disorders. Representative appearances of the eyes with SJS/TEN at before (J) and after 3-months use of the Limbal-Rigid CL (K,L).

steroids. Exposure of the corneal stroma can induce infectious or non-infectious corneal stromal thinning and perforation. Longlasting inflamed PEDs eventually result in symblepharon, as well as conjunctivalization and neovascularization of the cornea, which can lead to blindness.

In such severely inflamed eyes, limbal transplantation and amniotic membrane transplantation (AMT) do not guarantee epithelialization (13-15). Both cultivated corneal limbal epithelial transplantation (CLET) $(16,17)$ and cultivated oral mucosal epithelial sheet transplantation (COMET) (5) resulted in complete epithelialization of subacute PED in SJS/TEN cases, thus preventing end-stage cicatrization and vision loss. We hypothesize that one of the mechanisms by which COMET has a positive treatment effect on subacute PED is the decrease of massive inflammation on the ocular surface post surgery. While conventional limbal transplantation requires several weeks for the limbal epithelium from the donor cornea to migrate and cover the corneal surface, transplantation of cultivated epithelium covers the entire cornea during surgery and works to resolve the ocular surface inflammation.

\section{Management at the Chronic Phase}

In the chronic stage of SJS/TEN, ocular surface disease arising from the acute stage encompasses a spectrum of ocular manifestations and complications that are often associated with significant visual morbidity $(7,18,19)$. Visual impairment and ocular discomfort continue throughout the life of the patient, 
and usually require long-term medication for optimal control of the disease.

\section{Ocular Surface Grading Score}

To elucidate the profile of chronic ocular-disorder manifestations, we developed an OSGS as an objective method for grading the extent and severity of ocular complications in SJS/TEN (7). Ocular surface findings were

TABLE 1 | Univariate Logistic Regression Analysis Of The Association Between Variables At Onset And Acute Ocular Severity In The Patients with Stevens-Johnson syndrome (SJS) and toxic epidermal necrolysis (TEN) [modified version of the table presented in (4)].

\begin{tabular}{|c|c|c|c|}
\hline \multirow{2}{*}{$\begin{array}{l}\text { Variable at the onset } \\
n=135\end{array}$} & \multicolumn{3}{|c|}{ Univariate logistic regression } \\
\hline & OR & $95 \% \mathrm{Cl}$ & $P$-value \\
\hline Disease: TEN (vs. SJS) & 1.47 & $0.72-2.98$ & 0.287 \\
\hline $\begin{array}{l}\text { Gender: Male (vs. } \\
\text { Female) }\end{array}$ & 0.78 & $0.39-1.54$ & 0.466 \\
\hline Age at the onset (years) & 0.97 & $0.96-0.99$ & 0.004 \\
\hline $\begin{array}{l}\text { Age at onset (years): } \\
>50 \text { (vs. } 50 \leq \text { ) }\end{array}$ & 0.36 & $0.18-0.72$ & 0.004 \\
\hline NSAIDs & 2.04 & $1.02-4.1$ & 0.045 \\
\hline Cold-remedies & 5.51 & $1.72-17.62$ & 0.004 \\
\hline $\begin{array}{l}\text { NSAIDs or } \\
\text { cold-remedies }\end{array}$ & 2.68 & $1.33-5.38$ & 0.006 \\
\hline Antibiotics & 0.66 & $0.27-1.63$ & 0.363 \\
\hline Anticonvulsants & 0.72 & $0.33-1.58$ & 0.415 \\
\hline
\end{tabular}

$\mathrm{OR}$, odds ratio; $\mathrm{Cl}$, confidence interval; NSAIDs, non-steroidal anti-inflammatory drugs. classified as corneal complications (i.e., superficial punctate keratopathy, epithelial defect, loss of the palisades of Vogt, conjunctivalization, neovascularization, opacification, and keratinization), conjunctival complications (i.e., hyperemia, and symblepharon formation), and eyelid complications (trichiasis, mucocutaneous junction involvement, meibomian gland involvement, and punctal damage). In the OSGS, these 13 components are graded from 0 to 3 .

Among 138 SJS/TEN eyes treated, the most prevalent severe complications were loss of the palisades of Vogt (114 eyes, $82.6 \%$ ) and meibomian gland involvement (102 eyes, $73.9 \%$ ). Moreover, visual acuity in 74 of those 138 eyes (53.6 \%) was worse than 20/200. Eyes with a higher total score for the three complication categories had poorer vision $(R=0.806, p<$ $0.0001)$. Multivariable regression analysis showed that corneal neovascularization, opacification, keratinization, and cataracts significantly affected logMAR findings $(p<0.0001, p<0.0001, p$ $=0.0142$, and $p=0.0375$, respectively) (7).

Recently, our work using OSGS clearly demonstrated the long-term progression of ocular surface cicatrization in chronicphase SJS/TEN eyes, and in 35 (33.3\%) of 105 eyes, the total OSGS worsened during follow-up periods of over 5 years (20). Partial conjunctivalization progressed toward total conjunctivalization, and eyes with total conjunctivalization with partial keratinization progressed toward total keratinization. Thus, strict attention should be paid to eyes with partial conjunctivalization and partial keratinization.

\section{Management of the Ocular Surface}

In the management of chronic SJS/TEN cases, it is important to control ocular surface inflammation. In most cases, topical steroids are considered adequate treatment. However, since

TABLE 2 | Demographics of the Stevens-Johnson syndrome and toxic epidermal necrolysis cases seen at the acute and chronic phase.

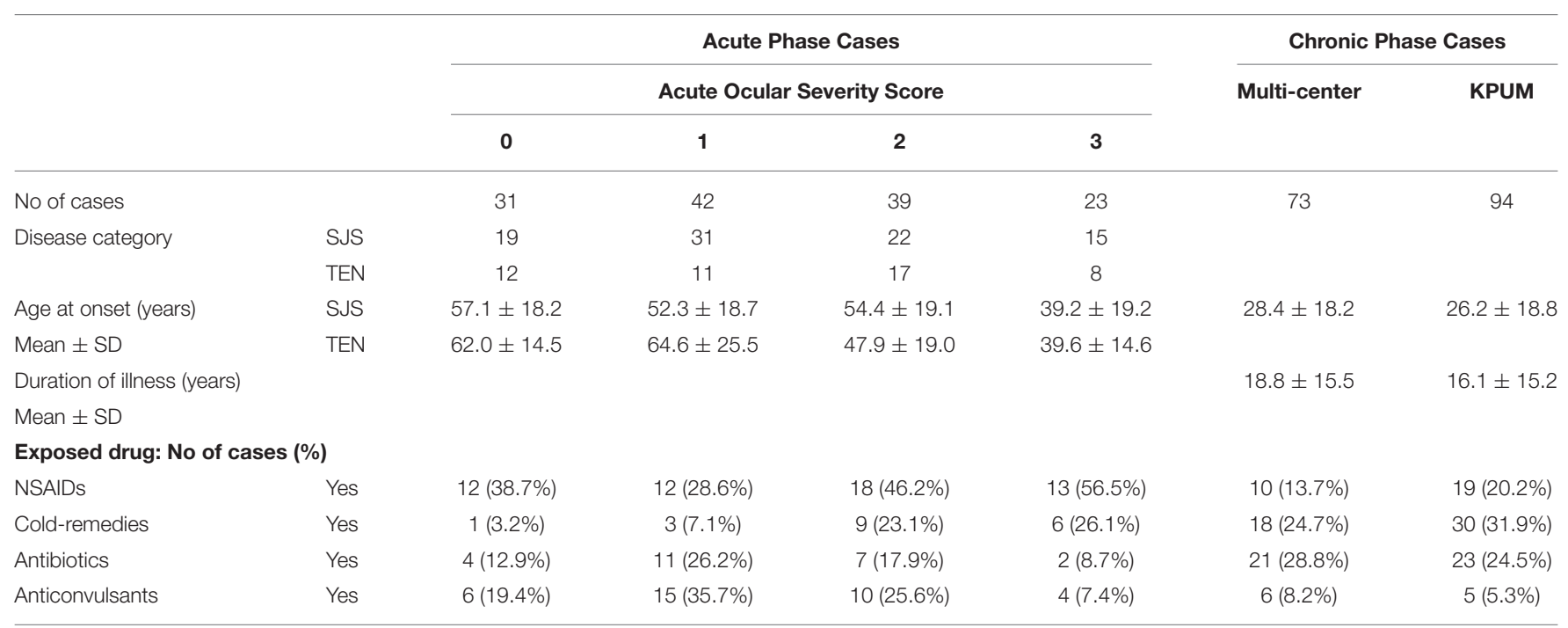

SJS, Stevens-Johnson syndrome; TEN, toxic epidermal necrolysis; SD, standard deviation; NSAIDs, non-steroidal anti-inflammatory drugs.

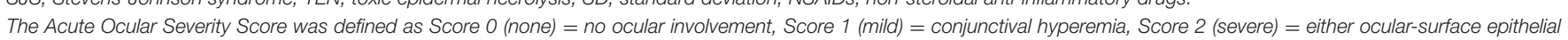

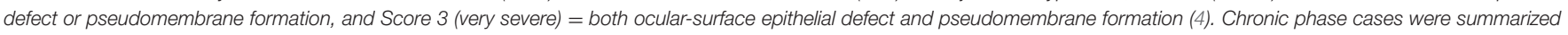
from our multi-center study in Japan (7) and our retrospective analysis for cases seen at Kyoto Prefectural University of Medicine (8). 
steroid-induced glaucoma can develop during the long-term use of topical steroids, strict attention should be paid to secondary glaucoma in SJS/TEN.

Decreasing the number of bacteria on the ocular surface is the key to obtaining complete stabilization of the ocular surface. Thus, appropriate use of topical antibiotics reduces the risk of ocular surface inflammation. Due to the high detection rate of methicillin-resistant Staphylococcus aureus (MRSA) and methicillin-resistant Staphylococcus epidermidis (MRSA), 0.5\% chloramphenicol is often prescribed, and $1 \%$ vancomycin eye ointment is known to be effective for MRSA/MRSE conjunctivitis or keratitis $(21,22)$.

Severe dry eye in SJS/TEN cases is comprised of three important mechanisms: (1) aqueous tear deficiency, (2) decreased wettability of the corneal surface, and (3) increased evaporation. The lacrimal punctum may be closed from scarring or cauterization, which can lead to a high tear meniscus and underestimation of the dry eye severity (23). Thus, it is important to suppress chronic inflammation on the ocular surface. The administration of $2 \%$ rebamipide ophthalmic solution reportedly helps to obtain ocular surface stabilization (24-26), and it often reduces or replaces topical steroid use.

\section{Surgical Interventions AMT}

In Japan, AMT was first performed in the mid 1990s $(27,28)$. Since then, AMT has been performed for recurrent pterygium, severe ocular surface disorders, and ocular surface neoplasia. Amniotic membrane is used after the release of symblepharon as the substrate of epithelial cells. However, in end-stage SJS/TEN cases, the effect of AMT is limited.

\section{COMET}

In 2002, we started performing COMET for the treatment of severe ocular disorders, including SJS/TEN (29-34). Our retrospective analysis of 86 COMET surgeries revealed that COMET is effective for visual improvement in eyes with chronic SJS/TEN (35). In end-stage cases with severe conjunctivalization, keratinization, and symblepharon, visual improvement was obtained, and the re-constructed ocular surface in those cases was maintained for a long time-period (Figures 1G-I) (36).

Based on the above results, a prospective clinical study of COMET for the treatment of severe ocular surface disorders (i.e., SJS, OCP, and severe thermal/chemical injury) was performed between September 2014 and March 2017 as part of a prospective clinical study under the Advanced Medical Care System in Japan. Thereafter, an investigator-initiated phase 3 clinical trial was started in 2018 to obtain regulatory approval for COMET. In both prospective clinical trials, COMET provided beneficial effects for the release of symblepharon and visual improvement (manuscript in preparation).

Post-surgical management is the key to obtaining favorable results. In our retrospective and prospective clinical studies, systemic corticosteroid (betamethasone, $1 \mathrm{mg} /$ day) and cyclosporine (2-3 $\mathrm{mg} / \mathrm{kg} / \mathrm{day})$ were administered to prevent postoperative inflammation and immunological response, and then tapered depending on the clinical findings. Nearly all patients required frequent administration of artificial tears, and a therapeutic soft contact lens was used for at least 1-month post surgery to protect the transplanted epithelium from mechanical ablation.

\section{Eye Lid Surgery}

Trichiasis is a common complication of SJS/TEN at the chronic stage. Double eyelashes and/or entropion are also seen in severely cicatrized cases. Eyelashes touching the cornea induce ocular surface inflammation, and might promote the deterioration of chronic SJS/TEN. Thus, it is optimal to correct these problems, and tarsal wedge resection, gray-line splitting or an autograft of hard-palate are considered depending on the severity of eyelid cicatrization. Interestingly, in a clinically "quiet" chronic SJS/TEN case, folliculitis reportedly existed (37). It should be noted that ocular surface stabilization can be obtained after the successful intervention of these surgeries.

\section{Visual Rehabilitation Using Limbal-Rigid Contact Lenses}

With the aim of reducing the symptoms related to corneal irregularity and dry eye observed in severe OSD cases, we developed a limbal-supported rigid-type CL (Limbal-Rigid CL; Sun Contact Lens Co., Inc., Kyoto, Japan) with a diameter ranging from 13 to $14 \mathrm{~mm}$. When our new CL is worn, a fluid layer exists at the peripheral zone of the lens, and the tears beneath the lens exchange at each blink.

A clinical study of the new Limbal-Rigid CL demonstrated significant improvement in VA and quality of life, particularly in SJS patients (38), and an investigator-initiated study for chronic SJS/TEN cases showed favorable results, the same as in the clinical study (Figures 1J-L) (39). We obtained regulatory approval for the Limbal-Rigid CL in 2016. Moreover, the LimbalRigid CL has good wettability, thus reducing eye pain related to severe dryness of the ocular surface, and it provides long-term maintenance of the ocular surface once stabilized.

It should be noted that use of the Limbal Rigid CL greatly enhances the postoperative outcome of COMET. The first step is COMET, followed by initiating Limbal Rigid CL wear (40).

\section{SUMMARY}

Ocular, oral, and nail manifestations are essential for a definitive diagnosis of SJS/TEN with ocular involvement. Acute conjunctivitis followed by skin eruptions with high fever indicates the initial sign of SJS/TEN, and early intervention with systemic and topical steroids at the acute stage appears to be important for the improvement of the visual prognosis. Although it remains impossible to fully restore the ocular surface to its normal healthy state (i.e., that of before disease onset), COMET alone, Limbal Rigid CL use alone, or the combination of both can greatly improve the vision and overall quality of life of patients with chronic-stage SJS/TEN. 


\section{AUTHOR CONTRIBUTIONS}

CS and MU drafted the manuscript. CS, MU, and SK revised the final version of the manuscript. All authors contributed to the article and approved the submitted version.

\section{FUNDING}

Supported in part by a Grant-in-Aid for Scientific Research from the Japanese Ministry of Health, Labour and Welfare (20FC1035), a Research Grant from the Japan Agency for Medical Research and Development (20ek0109377h), and a Research

\section{REFERENCES}

1. Bastuji-Garin S, Rzany B, Stern RS, Shear NH, Naldi L, Roujeau JC. Clinical classification of cases of toxic epidermal necrolysis, Stevens-Johnson syndrome, and erythema multiforme. Arch Dermatol. (1993) 129:92-6. doi: 10.1001/archderm.1993.016802201 04023

2. Roujeau JC. The spectrum of Stevens-Johnson syndrome and toxic epidermal necrolysis: a clinical classification. J Invest Dermatol. (1994) 102:28S-30S. doi: 10.1111/1523-1747.ep12388434

3. Auquier-Dunant A, Mockenhaupt M, Naldi L, Correia O, Schroder W, Roujeau JC. Correlations between clinical patterns and causes of erythema multiforme majus, Stevens-Johnson syndrome, and toxic epidermal necrolysis: results of an international prospective study. Arch Dermatol. (2002) 138:1019-24. doi: 10.1001/archderm.138.8.1019

4. Sotozono C, Ueta M, Nakatani E, Kitami A, Watanabe H, Sueki H, et al. Predictive factors associated with acute ocular involvement in StevensJohnson syndrome and toxic epidermal necrolysis. Am J Ophthalmol. (2015) 160:228-37 e2. doi: 10.1016/j.ajo.2015.05.002

5. Sotozono C, Inatomi T, Nakamura T, Koizumi N, Yokoi N, Ueta M, et al. Cultivated oral mucosal epithelial transplantation for persistent epithelial defect in severe ocular surface diseases with acute inflammatory activity. Acta Ophthalmol. (2014) 92:e447-53. doi: 10.1111/aos.12397

6. Di Pascuale MA, Espana EM, Liu DT, Kawakita T, Li W, Gao YY, et al. Correlation of corneal complications with eyelid cicatricial pathologies in patients with Stevens-Johnson syndrome and toxic epidermal necrolysis syndrome. Ophthalmology. (2005) 112:904-12. doi: 10.1016/j.ophtha.2004.11.035

7. Sotozono C, Ang LP, Koizumi N, Higashihara H, Ueta M, Inatomi T, et al. New grading system for the evaluation of chronic ocular manifestations in patients with Stevens-Johnson syndrome. Ophthalmology. (2007) 114:1294302. doi: 10.1016/j.ophtha.2006.10.029

8. Sotozono C, Ueta M, Koizumi N, Inatomi T, Shirakata $\mathrm{Y}$, Ikezawa $Z$, et al. Diagnosis and treatment of Stevens-Johnson syndrome and toxic epidermal necrolysis with ocular complications. Ophthalmology. (2009) 116:685-90. doi: 10.1016/j.ophtha.2008. 12.048

9. Roujeau JC, Bastuji-Garin S. Systematic review of treatments for StevensJohnson syndrome and toxic epidermal necrolysis using the SCORTEN score as a tool for evaluating mortality. Ther Adv Drug Saf. (2011) 2:8794. doi: 10.1177/2042098611404094

10. Kim KH, Park SW, Kim MK, Wee WR. Effect of age and early intervention with a systemic steroid, intravenous immunoglobulin or amniotic membrane transplantation on the ocular outcomes of patients with Stevens-Johnson syndrome. Korean J Ophthalmol. (2013) 27:33140. doi: 10.3341/kjo.2013.27.5.331

11. Araki $\mathrm{Y}$, Sotozono $\mathrm{C}$, Inatomi $\mathrm{T}$, Ueta $\mathrm{M}$, Yokoi $\mathrm{N}$, Ueda $\mathrm{E}$, et al. Successful treatment of Stevens-Johnson syndrome with steroid pulse therapy at disease onset. Am J Ophthalmol. (2009) 147:1004-11:11 e1. doi: 10.1016/j.ajo.2008.12.040
Grant from the Japanese Ministry of Education, Culture, Sports, Science and Technology (19H03809).

\section{ACKNOWLEDGMENTS}

We would like to thank for the Japanese Research Committee on Severe Cutaneous Adverse reaction for their understanding of the ocular sequelae caused from SJS/TEN, and also thank for Mr. Masanori Fukushima and the Foundation for Biomedical Research and Innovation, at Kobe, Kobe, Japan, for their continuous support to translational research of COMET.

12. Yagi T, Sotozono C, Tanaka M, Fuwa M, Sekiyama E, Ueta M, et al. Cytokine storm arising on the ocular surface in a patient with Stevens-Johnson syndrome. Br J Ophthalmol. (2011) 95:1030-1. doi: 10.1136/bjo.2010.196295

13. Azuara-Blanco A, Pillai CT, Dua HS. Amniotic membrane transplantation for ocular surface reconstruction. Br J Ophthalmol. (1999) 83:399402. doi: 10.1136/bjo.83.4.399

14. Tamhane A, Vajpayee RB, Biswas NR, Pandey RM, Sharma N, Titiyal JS, et al. Evaluation of amniotic membrane transplantation as an adjunct to medical therapy as compared with medical therapy alone in acute ocular burns. Ophthalmology. (2005) 112:1963-9. doi: 10.1016/j.ophtha.2005.05.022

15. Saw VP, Minassian D, Dart JK, Ramsay A, Henderson H, Poniatowski S, et al. Amniotic membrane transplantation for ocular disease: a review of the first 233 cases from the UK user group. Br J Ophthalmol. (2007) 91:10427. doi: $10.1136 /$ bjo.2006.098525

16. Koizumi N, Inatomi T, Suzuki T, Sotozono C, Kinoshita S. Cultivated corneal epithelial stem cell transplantation in ocular surface disorders. Ophthalmology. (2001) 108:1569-74. doi: 10.1016/S0161-6420(01)00694-7

17. Kinoshita S, Koizumi N, Sotozono C, Yamada J, Nakamura T, Inatomi T. Concept and clinical application of cultivated epithelial transplantation for ocular surface disorders. Ocul Surf. (2004) 2:21-33. doi: 10.1016/S1542-0124(12)70021-6

18. Power WJ, Ghoraishi M, Merayo-Lloves J, Neves RA, Foster CS. Analysis of the acute ophthalmic manifestations of the erythema multiforme/StevensJohnson syndrome/toxic epidermal necrolysis disease spectrum. Ophthalmology. (1995) 102:1669-76. doi: 10.1016/S0161-6420(95)30811-1

19. Kawasaki S, Nishida K, Sotozono C, Quantock AJ, Kinoshita S. Conjunctival inflammation in the chronic phase of Stevens-Johnson syndrome. $\mathrm{Br} \mathrm{J}$ Ophthalmol. (2000) 84:1191-3. doi: 10.1136/bjo.84.10.1191

20. Yoshikawa Y, Ueta M, Nishigaki H, Kinoshita S, Ikeda T, Sotozono C. Predictive biomarkers for the progression of ocular complications in chronic Stevens-Johnson syndrome and toxic epidermal necrolysis. Sci Rep. (2020) 10:18922. doi: 10.1038/s41598-020-76064-8

21. Sotozono C, Inagaki K, Fujita A, Koizumi N, Sano $\mathrm{Y}$, Inatomi $\mathrm{T}$, et al. Methicillin-resistant Staphylococcus aureus and methicillin-resistant Staphylococcus epidermidis infections in the cornea. Cornea. (2002) 21(7 Suppl.):S94-101. doi: 10.1097/01.ico.0000263127.84015.3f

22. Sotozono C, Fukuda M, Ohishi M, Yano K, Origasa H, Saiki $\mathrm{Y}$, et al. Vancomycin Ophthalmic Ointment $1 \%$ for methicillinresistant Staphylococcus aureus or methicillin-resistant Staphylococcus epidermidis infections: a case series. BMJ Open. (2013) 3:e001206. doi: 10.1136/bmjopen-2012-001206

23. Sotozono C, Ueta M, Yokoi N. Severe dry eye with combined mechanisms is involved in the ocular sequelae of SIS/TEN at the chronic stage. Invest Ophthalmol Vis Sci. (2018) 59:DES80-6. doi: 10.1167/iovs.18-24019

24. Kinoshita S, Awamura S, Nakamichi N, Suzuki H, Oshiden K, Yokoi N. A multicenter, open-label, 52-week study of 2\% rebamipide (OPC-12759) ophthalmic suspension in patients with dry eye. Am J Ophthalmol. (2014) 157:576-83 e1. doi: 10.1016/j.ajo.2013.11.010

25. Kinoshita S, Oshiden K, Awamura S, Suzuki H, Nakamichi N, Yokoi N. A randomized, multicenter phase 3 study comparing $2 \%$ rebamipide 
(OPC-12759) with $0.1 \%$ sodium hyaluronate in the treatment of dry eye. Ophthalmology. (2013) 120:1158-65. doi: 10.1016/j.ophtha.2012.12.022

26. Ueta M, Sotozono C, Yokoi N, Kinoshita S. Rebamipide suppresses PolyI:Cstimulated cytokine production in human conjunctival epithelial cells. J Ocul Pharmacol Ther. (2013) 29:688-93. doi: 10.1089/jop.2012.0054

27. Tsubota K, Satake Y, Ohyama M, Toda I, Takano Y, Ono M, et al. Surgical reconstruction of the ocular surface in advanced ocular cicatricial pemphigoid and Stevens-Johnson syndrome. Am J Ophthalmol. (1996) 122:38-52. doi: 10.1016/S0002-9394(14)70510-0

28. Shimazaki J, Yang HY, Tsubota K. Amniotic membrane transplantation for ocular surface reconstruction in patients with chemical and thermal burns. Ophthalmology. (1997) 104:2068-76. doi: 10.1016/S0161-6420(97)30057-8

29. Nakamura T, Endo K, Cooper LJ, Fullwood NJ, Tanifuji N, Tsuzuki M, et al. The successful culture and autologous transplantation of rabbit oral mucosal epithelial cells on amniotic membrane. Invest Ophthalmol Vis Sci. (2003) 44:106-16. doi: 10.1167/iovs.02-0195

30. Nakamura T, Inatomi T, Sotozono C, Amemiya T, Kanamura N, Kinoshita S. Transplantation of cultivated autologous oral mucosal epithelial cells in patients with severe ocular surface disorders. Br J Ophthalmol. (2004) 88:1280-4. doi: 10.1136/bjo.2003.038497

31. Inatomi $\mathrm{T}$, Nakamura $\mathrm{T}$, Koizumi $\mathrm{N}$, Sotozono $\mathrm{C}$, Yokoi $\mathrm{N}$, Kinoshita $\mathrm{S}$. Midterm results on ocular surface reconstruction using cultivated autologous oral mucosal epithelial transplantation. Am J Ophthalmol. (2006) 141:26775. doi: 10.1016/j.ajo.2005.09.003

32. Inatomi T, Nakamura $\mathrm{T}$, Kojyo M, Koizumi N, Sotozono C, Kinoshita S. Ocular surface reconstruction with combination of cultivated autologous oral mucosal epithelial transplantation and penetrating keratoplasty. Am J Ophthalmol. (2006) 142:757-64. doi: 10.1016/j.ajo.2006.06.004

33. Ang LP, Nakamura T, Inatomi T, Sotozono C, Koizumi N, Yokoi N, et al. Autologous serum-derived cultivated oral epithelial transplants for severe ocular surface disease. Arch Ophthalmol. (2006) 124:154351. doi: 10.1001/archopht.124.11.1543

34. Nakamura T, Kinoshita S. Ocular surface reconstruction using cultivated mucosal epithelial stem cells. Cornea. (2003) 22(7 Suppl.):S75-80. doi: 10.1097/00003226-20031000100011

35. Sotozono C, Inatomi T, Nakamura T, Koizumi N, Yokoi N, Ueta M, et al. Visual improvement after cultivated oral mucosal epithelial transplantation. Ophthalmology. (2013) 120:193-200. doi: 10.1016/j.ophtha.2012. 07.053
36. Nakamura T, Takeda K, Inatomi T, Sotozono C, Kinoshita S. Long-term results of autologous cultivated oral mucosal epithelial transplantation in the scar phase of severe ocular surface disorders. Br J Ophthalmol. (2011) 95:942-6. doi: 10.1136/bjo.2010.188714

37. Watanabe A, Sotozono C, Ueta M, Shinomiya K, Kinoshita S, Kakizaki H, et al. Folliculitis in clinically "quiet" chronic StevensJohnson syndrome. Ophthalmic Plast Reconstr Surg. (2014) 30:80-2. doi: 10.1097/01.iop.0000440709.90516.87

38. Sotozono C, Yamauchi N, Maeda S, Kinoshita S. Tear exchangeable limbal rigid contact lens for ocular sequelae resulting from Stevens-Johnson syndrome or toxic epidermal necrolysis. Am J Ophthalmol. (2014) 158:98393. doi: 10.1016/j.ajo.2014.07.012

39. Itoi M, Ueta M, Ogino K, Sumi E, Imai K, Teramukai S, et al. Clinical trial to evaluate the therapeutic benefits of limbal-supported contact lens wear for ocular sequelae due to Stevens-Johnson syndrome/toxic epidermal necrolysis. Cont Lens Anterior Eye. (2020) 43:535-42. doi: 10.1016/j.clae.2020. 05.007

40. Sotozono C, Inatomi T, Nakamura T, Ueta M, Imai K, Fukuoka H, et al. Oral mucosal epithelial transplantation and limbal-rigid contact lens: a therapeutic modality for the treatment of severe ocular surface disorders. Cornea. (2020) 39(Suppl. 1):S19-27. doi: 10.1097/ICO.0000000000002566

Conflict of Interest: The authors declare that the research was conducted in the absence of any commercial or financial relationships that could be construed as a potential conflict of interest.

Publisher's Note: All claims expressed in this article are solely those of the authors and do not necessarily represent those of their affiliated organizations, or those of the publisher, the editors and the reviewers. Any product that may be evaluated in this article, or claim that may be made by its manufacturer, is not guaranteed or endorsed by the publisher.

Copyright (c) 2021 Sotozono, Ueta and Kinoshita. This is an open-access article distributed under the terms of the Creative Commons Attribution License (CC BY). The use, distribution or reproduction in other forums is permitted, provided the original author(s) and the copyright owner(s) are credited and that the original publication in this journal is cited, in accordance with accepted academic practice. No use, distribution or reproduction is permitted which does not comply with these terms. 